\title{
Capture of mesothelioma cells with 'universal' CTC-chip
}

\author{
KAZUE YONEDA $^{1}$, YASUHIRO CHIKAISHI ${ }^{1}$, TAIJI KUWATA ${ }^{1}$, TAKASHI OHNAGA ${ }^{2}$ and FUMIHIRO TANAKA ${ }^{1}$ \\ ${ }^{1}$ Second Department of Surgery, University of Occupational and Environmental Health, Kitakyushu, \\ Fukuoka 807-8555; ${ }^{2}$ Central Research Institute, Toyama Industrial Technology Center, Takaoka, Toyama 933-0981, Japan
}

Received March 14, 2017; Accepted September 12, 2017

DOI: 10.3892/ol.2017.7619

\begin{abstract}
Malignant mesothelioma (MM) is a highly aggressive malignant tumor, predominantly associated with job-related exposure to asbestos. Development of effective and non-invasive modalities for diagnosis is an important issue in occupational medicine. Circulating tumor cells (CTCs), which are tumor cells that are shed from primary tumors and circulate in the peripheral blood, may be detected at an earlier stage than malignant tumors, and detection of CTCs may provide a novel insight into the diagnosis of MM. In a previous study evaluating clinical utility of CTCs, detected with a widely used system 'CellSearch', the authors indicated a significant however insufficient capability in the diagnosis of MM, suggesting need for a more sensitive system. Accordingly, the authors developed a novel microfluidic system to capture CTCs (CTC-chip), and demonstrated that the CTC-chip effectively captured MM cells (ACC-MESO-4) spiked in the blood by conjugating an anti-podoplanin antibody. The results of the present study demonstrated that the CTC-chip coated with the anti-podoplanin antibody captured another MM cell (ACC-MESO-1). However, the capture efficiencies were lower than those for ACC-MESO-4. In addition, an anti-mesothelin antibody was used to capture CTCs, however the CTC-chip coated with the anti-mesothelin antibody failed to effectively capture MM cells, possibly due to low mesothelin expression. Overall, the CTC-chip may capture specific types of CTCs by conjugating any antibody against an antigen expressed on CTCs, and may be a useful system for the diagnosis of malignant tumors, including MM.
\end{abstract}

\section{Introduction}

Malignant mesothelioma (MM) is a highly aggressive tumor of the mesothelial origin associated with asbestos exposure, and most commonly develops in the pleura (1-3). MM is rare,

Correspondence to: Dr Kazue Yoneda, Second Department of Surgery, University of Occupational and Environmental Health, 1-1 Iseigaoka, Yahatanishi-ku, Kitakyushu, Fukuoka 807-8555, Japan E-mail: yoneda@med.uoeh-u.ac.jp

Key words: mesothelioma, circulating tumor cells, CTC-chip, epithelial cell adhesion molecule, podoplanin, mesothelin but is increasingly prevalent in many industrialized countries including Japan even after a ban of asbestos usage probably because of its long latency period between asbestos exposure and development of MM (3-5). Thus, the prevention, diagnosis and therapy of MM are important issues in occupational medicine.

The diagnosis of MM is usually confirmed by histological examination of biopsied samples, which are usually obtained invasive procedures such as thoracoscopic pleural biopsy $(6,7)$. These invasive procedures may not be appropriate for mass-screening to identify MM patients among high-risk population with history of asbestos-exposure, or cannot be performed for patients with impaired organ functions. Among less invasive procedures for the diagnosis, radiographic examinations such as chest roentgenogram and computed tomography (CT) are most commonly employed, but do not provide definitive diagnosis of MM. Blood-based tests may be promising, but the serum mesothelin related protein (SMRP), the only clinically approved blood-test, may not provide sufficient diagnostic sensitivity $(8,9)$. Accordingly, a novel blood-based test for the diagnosis of MM should be established for early diagnosis as well as improvement of prognosis of MM patients.

Circulating tumor cells (CTCs) are tumor cells that are shed from the primary tumor and circulate in the peripheral blood (10). CTCs may be promising marker as a surrogate of micro-metastasis, but detection of rare tumor cells contaminated in a vast majority of normal hematological cells may present a technical challenge $(10,11)$. The 'CellSearch' system (Veridex LCC, Raritan, NJ, USA) is an automated detection system of CTCs using an antibody against an epithelial marker (EpCAM), which is the only approved system for the clinical use (only in USA) (12). In a previous study, we evaluated CTCs with the 'CellSearch' in peripheral blood sampled from patients with diagnosis or suspicion of MM. The CTC-test provided a significant prognostic value in discrimination between MM patients and non-MM patients such as asbestos pleurisy $(\mathrm{P}=0.036)$, but the sensitivity was only modest $(32.7 \%)$ mainly due to negative or low expression of EpCAM on MM cells, which may not be effectively captured with an anti-EpCAM antibody (13). These results clearly indicate the need for a sensitive system for capture EpCAM-negative CTCs, and we have developed a high efficient system to capture CTCs using a microfluidic device 'CTC-chip' $(14,15)$. In the system, CTCs are captured to numerous micro-posts coated with an antibody against an antigen expressed on target tumor cells, 
and the most important advantage is capability of conjugating any antibody to capture CTCs. In fact, we effectively captured and isolated EpCAM-negative MM cells (ACC-MESO-4 cells) with the CTC-chip coated with an antibody against a mesothelial marker (podoplanin) (15), indicating its potential capability of capturing a wide variety of CTCs by conjugating appropriate capture antibodies. In the current study, we expand and examined capture efficiencies of the 'universal' CTC-chip for another MM cell-line (ACC-MESO-1) and with another capture antibody against another mesothelial marker (mesothelin) to improve sensitivity in detection of CTCs for clinical application in the diagnosis of MM.

\section{Materials and methods}

Cell lines. Human mesothelioma cell lines, ACC-MESO-1 and ACC-MESO-4 established in Aichi Cancer Research Center (16) as well as a human lung adenocarcinoma cell line, PC-9, were purchased from Riken BioResource Center (Tsukuba, Japan). These cells ware cultured in RPMI-1640 medium (Wako Pure Chemical Industries, Osaka, Japan) supplemented with $10 \%$ fetal bovine serum (Invitrogen; Thermo Fisher Scientific, Inc., Waltham, MA, USA) at $37^{\circ} \mathrm{C}$ and $5 \% \mathrm{CO}_{2}$.

Flow cytometry. Cells were collected and incubated with a primary antibody, an anti-EpCAM antibory (clone HEA125; Santa Cruz Biotechnology, Inc., Dallas, TX, USA), an anti-podoplanin antibody (clone E1; Santa Cruz Biotechnology, Inc.), or an anti-mesothelin antibody (clone K1; Santa Cruz Biotechnology, Inc.). Then, cells were incubated with a goat anti-mouse IgG antibody conjugated with FITC (BD Biosciences, San Jose, CA, USA). Flow cytometry analysis was performed using EC800 Cell Analyser (Sony Biotechnology, Tokyo, Japan) and FlowJo software (Tree Star, Inc., Ashland,. OR, USA). The percentage of positive cells and the mean fluorescence intensity (MFI) was determined by comparison with negative control.

Preparation of CTC-chip. The polymeric CTC-chip system was used after two-step coating with an antibody to capture CTCs as described previously (15). In brief, the chip was first incubated with a goat anti-mouse $\mathrm{IgG}$ antibody (SouthernBiotech, Birmingham, AL, USA), and then was incubated with an anti-EpCAM antibody (clone HEA125), an anti-podoplanin antibody, or an anti-mesothelin antibody (clone K1) to capture tumor cells; the antibody-coated chip was referred to as 'EpCAM-chip', 'podoplanin-chip', and 'mesothelin-chip', respectively. After washing with PBS, the chip surface was kept wet.

Sample preparation and evaluation of cell-capture efficacy. Sample preparation and flow test were performed as described previously (15). In brief, 500 tumor cells, labeled with CellTrace $^{\mathrm{TM}}$ CFSE Cell Proliferation Kit (Thermo Fisher Scientific, Inc.) and suspended in $1 \mathrm{ml}$ of phosphate-buffered saline (PBS) containing 5\% BSA were applied to the CTC-chip system.

Images and movies of cells in the chip were monitored and recorded with a fluorescence microscope CKX41 (Olympus
Corporation, Tokyo, Japan) and a digital video camera (Sony Biotechnology), and determined the actual number of cells (N-total) that were sent into the chip by counting the number of cells that passed through the inlet of the chip as well as the number of captured cells (N-captured) by counting CFSE-labelled cells remained on the chip. The cell capture efficiency was represented as $\mathrm{N}$-captured/N-total $(14,15)$. The average and standard error (SE) of capture efficiency were calculated from results obtained in triplicated experiments. Data were compared using a non-parametric test (Mann-Whitney U-test for comparison between 2 groups or Kruskal-Wallis H-test for comparison among 3 groups). $\mathrm{P}<0.05$ was considered to indicate a statistically significant difference. All statistical analyses were performed with the SPSS software package (version 21.0; IBM Corp., Armonk, NY, USA).

The study was reviewed and approved by the Institutional Review Board of the University of Occupational and Environmental Health, Japan.

\section{Results}

Expression of EpCAM and podoplanin on ACC-MESO-1 cells. ACC-MESO-1, a human MM cell line, did not express EpCAM and modestly expressed podoplanin (Fig. 1A).

Expression of Mesothelin on PC-9, ACC-MESO-1, ACC-MESO-4 cells. PC-9, a human lung adenocarcinoma cell, did not express mesothelin, and ACC-MESO-1 and MESO-4, human MM cell lines, weakly expressed mesothelin (Fig. 1B).

Cell capture efficiency. When ACC-MESO-1 cells were spiked in PBS, cells were not captured by the EpCAM-chip (average capture efficiency, $3.5 \%$ ); cells were captured with the podoplanin chip (Fig. 2, Table I. $\mathrm{P}=0.005$ ), but the average capture efficiency was only modest (average capture efficiency, 52.7\%) as compared with that $(78.3 \%)$ for ACC-MESO-4 obtained in the previous study (15). The average captures efficiency of the mesothelin-chip for PC-9, ACC-MESO-1, and ACC-MESO-4 were 2.9, 4.3 and 5.4\%, respectively (Fig. 3, Table II).

When ACC-MESO-1 cells were spiked in PBS, cells were captured by the podoplanin-chip (average capture efficiency, $52.7 \%$ ). It was significantly higher than with the EpCAM chip, the average capture efficiency was $3.5 \%$ (Fig. 2, Table I. $\mathrm{P}=0.005$, calculated by Mann-Whitney U-test), but the average capture efficiency was only modest (average capture efficiency, $52.7 \%)$ as compared with that $(78.3 \%)$ for ACC-MESO-4 obtained in the previous study (15). The average captures efficiency of the mesothelin-chip for PC-9, ACC-MESO-1, and ACC-MESO-4 were 2.9, 4.3, and 5.4\%, respectively (Fig. 3, Table II. $\mathrm{P}=0.329$, calculated by Kruskal-Wallis H-test).

\section{Discussion}

In the present study, we showed that the novel CTC-chip can capture mesothelioma cells (ACC-MESO-1) when coated with an antibody against podoplanin that is a specific antigen expressed on the surface of mesothelial cells (17-19). In the previous study, we showed that the 'podoplanin-chip' effectively captured mesothelioma cells of another cell line, 

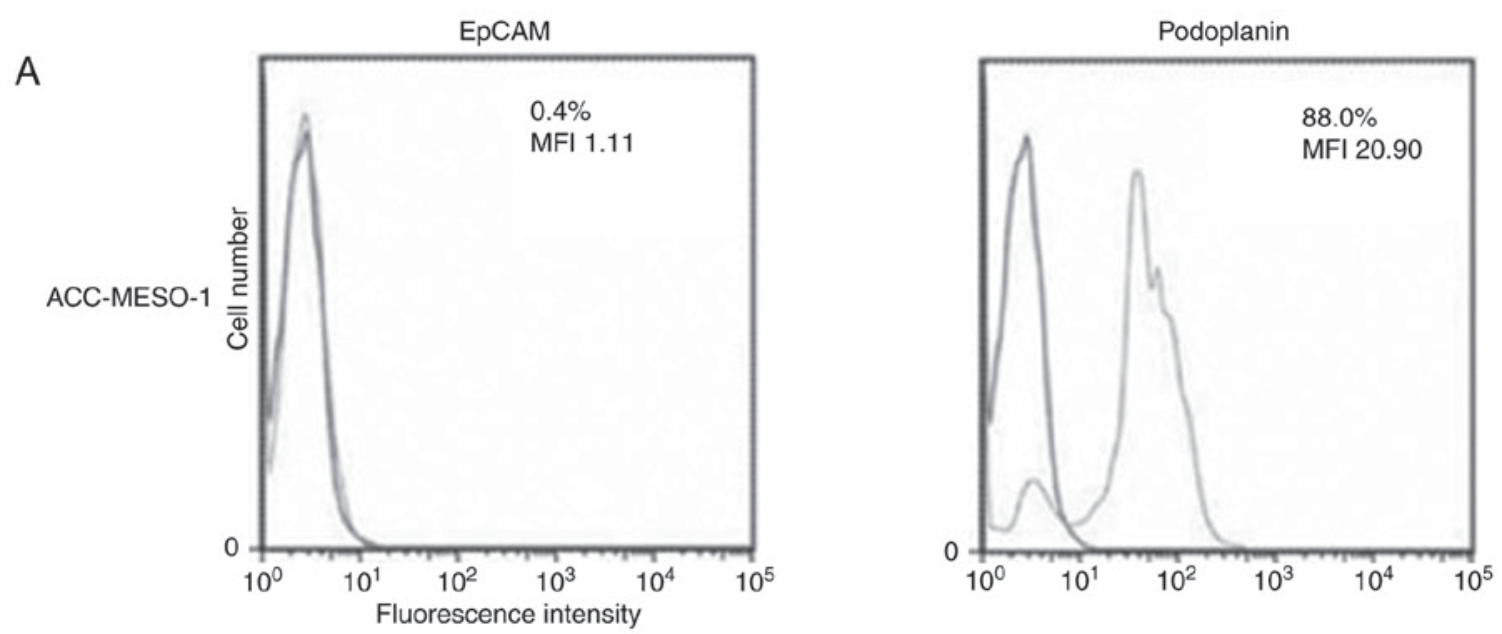

B
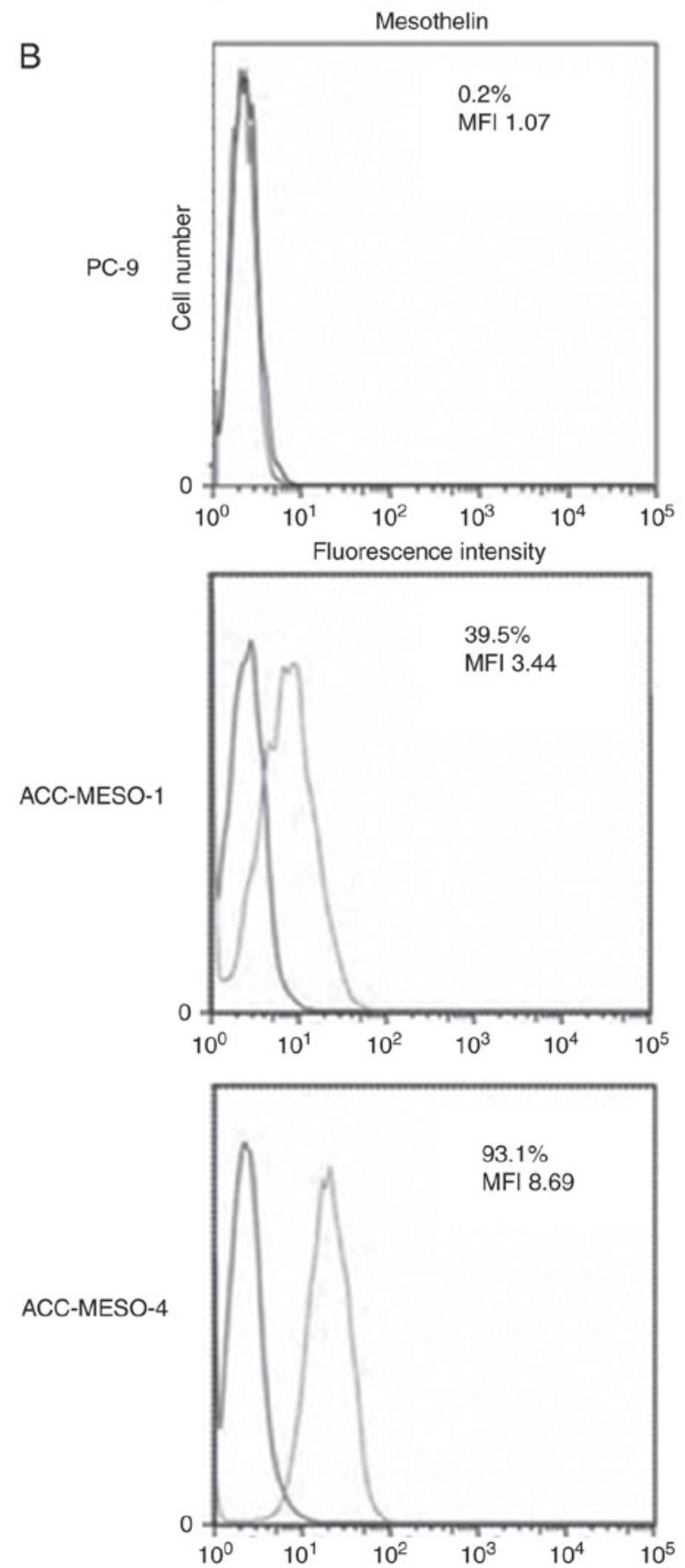

Figure 1. Flowcytometry analysis of cell surface antigen. EpCAM and podoplanin expression on ACC-MESO-1 cells (A). Mesothelin expression on PC-9, ACC-MESO-1, ACC-MESO-4 cells (B). 


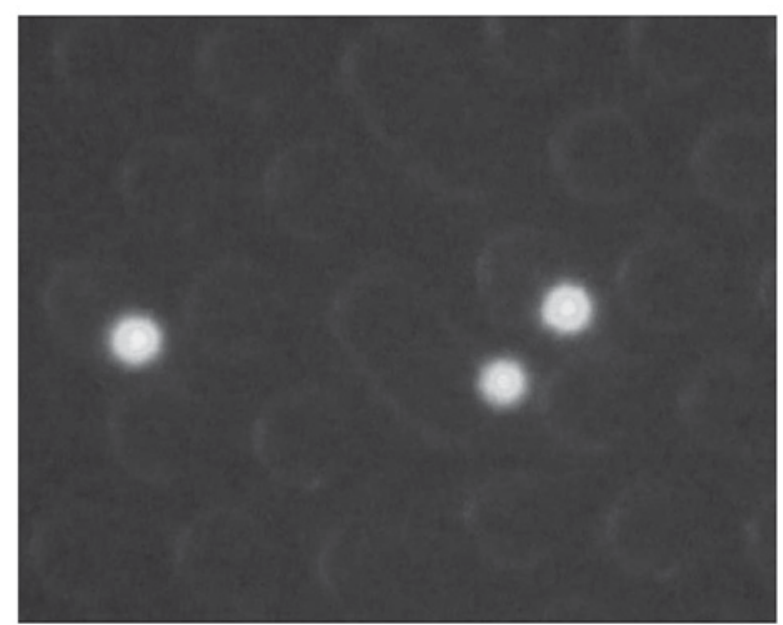

Figure 2. Captured ACC-MESO-1 cells with the 'podoplanin-chip'.

ACC-MESO-4 (15), indicating that the novel CTC-chip is a promising modality to detect some kinds of tumor cells including EpCAM-negative cells due to non-epithelial origin (e.g., mesothelioma cells) or undergoing epithelial-mesenchymal transition (EMT). Among a variety of systems for capture EpCAM-negative CTCs such as size-based or density-based separation systems (20), we employed a microfluidic system to capture CTCs called CTC-chip $(14,15)$. The CTC-chip had been originally developed in USA (21), but the original CTC-chip can capture only EpCAM-positive CTCs because an anti-EpCAM antibody to capture CTCs is conjugated and another antibody is not available in the system. In contrast, the novel CTC-chip can be easily conjugated with any antibody, and can capture a variety of CTCs regardless of EpCAM expression status, which referred as a 'universal' CTC-chip (15). In fact, as shown in this study, the CTC-chip can actually capture EpCAM-negative and podoplanin-positive mesothelioma cell (ACC-MESO-1) when conjugated with an anti-podoplanin antibody, and can capture EpCAM-positive PC-9 cells when conjugated with an anti-EpCAM antibody (15).

The current study showed a modest efficacy of the podoplanin chip to capture ACC-MESO-1 cells spiked in PBS with an average capture efficiency of $52.7 \%$, which was somewhat lower than that for ACC-MESO-4 (average capture rate, 78.3\%) obtained in the previous study (15). In the previous study, the average capture efficiency of the EpCAM-chip to capture PC-9 cells with strongest EpCAM expression was $100 \%$ and still higher than that $(78.3 \%)$ of the podoplnin-chip to capture ACC-MESO-4 cells with enhanced podoplanin expression, indicating that the capture efficiency might be influenced antigen-antibody interaction caused mainly by degree of antigen expression on the surface of tumor cells (15). The current study also showed that the capture of ACC-MESO-1 cells with modest podoplanin expression was less effective by the 'podoplanin-chip' (Table III). We also tried to capture another mesothelioma cell line, MSTO-211H derived from biphasic mesothelioma patient, with CTC-chip coated with the anti-podoplanin antibody (clone E1). However it could not capture well $(8.3 \%, \mathrm{~N}$-captured/N-total; 51/611), because $211 \mathrm{H}$ cells did not express podoplanin (data not shown). We have 
Table II. Capture from the cell suspension spiked in PBS with 'Mesothelin-chip'.

\begin{tabular}{|c|c|c|c|c|c|}
\hline \multirow[b]{3}{*}{ Variable } & \multicolumn{5}{|c|}{ Mesothelin-chip } \\
\hline & \multirow{2}{*}{$\begin{array}{c}\text { No. of cells } \\
\text { captured } \\
\text { (N-captured) }\end{array}$} & \multirow{2}{*}{$\begin{array}{l}\text { No. of } \\
\text { total cells } \\
\text { (N-total) }\end{array}$} & \multicolumn{2}{|c|}{$\begin{array}{c}\text { Cell capture } \\
\text { efficiency }(\%)\end{array}$} & \multirow{2}{*}{$\begin{array}{l}\text { Standard } \\
\text { Error } \\
(\mathrm{SE})\end{array}$} \\
\hline & & & Values & Average & \\
\hline \multirow[t]{3}{*}{ PC-9 } & 6 & 535 & 1.1 & 2.9 & \\
\hline & 17 & 518 & 3.3 & & 0.95 \\
\hline & 27 & 632 & 4.3 & & \\
\hline \multirow[t]{3}{*}{ ACC-MESO-1 } & 37 & 706 & 5.2 & 4.3 & \\
\hline & 28 & 614 & 4.6 & & 0.66 \\
\hline & 23 & 762 & 3.0 & & \\
\hline \multirow[t]{3}{*}{ ACC-MESO-4 } & 38 & 591 & 6.4 & 5.4 & \\
\hline & 15 & 469 & 3.2 & & 1.12 \\
\hline & 50 & 749 & 6.7 & & \\
\hline
\end{tabular}

EpCAM, epithelial cell adhesion molecule. $\mathrm{P}=0.329$ (Kruskal-Wallis H-test).

Table III. Capture efficiency of tumor cells spiked in PBS.

\begin{tabular}{lrcc}
\hline Variable & PC-9 & $\begin{array}{c}\text { ACC- } \\
\text { MESO-1 }\end{array}$ & $\begin{array}{c}\text { ACC- } \\
\text { MESO-4 }\end{array}$ \\
\hline EpCAM-chip & $101.1^{\mathrm{a}}$ & 3.5 & $2.3^{\mathrm{a}}$ \\
Podoplanin-chip & $3.0^{\mathrm{a}}$ & 52.0 & $78.3^{\mathrm{a}}$ \\
Mesothelin-chip & 2.9 & 4.3 & 5.4 \\
\hline
\end{tabular}

EpCAM, epithelial cell adhesion molecule. ${ }^{a}$ Data for PC-9 and ACC-MESO-4 were obtained in previous experiment and presented in a previous manuscript (ref. 15).

to try other MM cells in the future. For clinical samples, it is under consideration about optimal detection methods.

In the current study, we employed and examined a novel antibody, an anti-mesothelin antibody clone K1, to capture MM cells as mesothelin is one of useful biomarkers of MM (22). However, the 'mesothelin-chip' failed to provide effective capture performance (Fig. 3B), mainly due to lower mesothelin expression on MM cells (Fig. 1B). We tried to capture MSTO-211H cells using CTC-chip coated with anti-mesothelin antibody clone K1. Mesothelin expression of MSTO-211H cells were measured by flow cytometry and compared with ACC-MESO-4 cells as positive control. A sample without the primary antibody was used as negative control. Consequently, the mesothelin expression of MSTO-211H cells were almost negative (data not shown), it was in consistent to the previous report (23), and these cells failed to capture effectively using 'mesothelin-chip'. The capture efficiency of MSTO-211H cells was 6.2\% (28/453) (data not shown). In addition, we tried to capture using another anti-mesothelin antibody, clone 5B2 mouse monoclonal antibody. For ACC-MESO-1 or ACC-MESO-4 cells, capture efficiency were $14.3 \%(12 / 84)$, $7.3 \%(6 / 82)$ respectively (data not shown). They were hardly

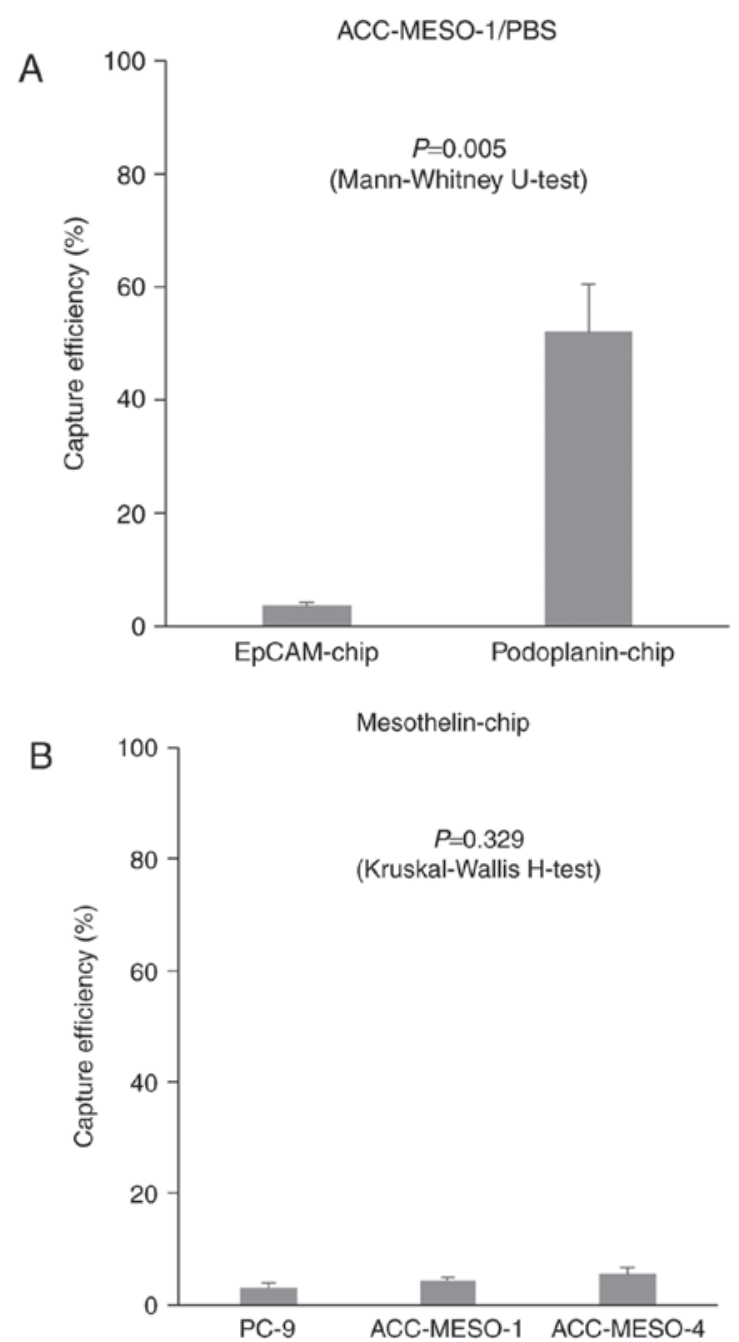

Figure 3. Capture efficiency of the 'CTC-chip' coated with various antibody to capture tumor cells. Mean $\pm \mathrm{SE}$ of three experiments. Capture efficiency of the 'EpCAM-chip' or 'Podoplanin-chip' when (A) ACC-MESO-1 cells spiked in PBS. Capture efficiency of the 'Mesothelin-chip' when (B) PC-9, ACC-MESO-1 or ACC-MESO-4 cells were spiked in PBS. 
captured as well as anti-mesothelin antibody clone K1 (MESO-1: 3.0-5.2\%, MESO-4: 3.2-6.7\%). It is need to examine by obtaining other cell lines more expressed mesothelin or using other mesothelin antibody. In future studies, other antibodies such as an antibody against CD146, a recently identified mesothelial marker (24), will be tested to capture MM cells. In conclusion, the 'universal' CTC-chip might be a useful system as less-invasive blood-based modality in the diagnosis of MM.

\section{Acknowledgements}

The authors would like to thank Eri Kawashima for her technical assistance. This study was supported Grants-in-Aid for Scientific Research (Grant nos. 26861131, and 16K10697) from the Japan Society for the Promotion of Science (JSPS) and by the Grant of The Clinical Research Promotion Foundation 2015.

\section{References}

1. Ismail-Khan R, Robinson LA, Williams CC Jr, Garrett CR, Bepler G and Simon GR: Malignant pleural mesothelioma: A comprehensive review. Cancer Control 13: 255-263, 2006.

2. Tsiouris A and Walesby RK: Malignant pleural mesothelioma: Current concepts in treatment. Nat Clin Pract Oncol 4: 344-352, 2007.

3. Lin RT, Takahashi K, Karjalainen A, Hoshuyama T, Wilson D, Kameda T, Chan CC, Wen CP, Furuya S, Higashi T, et al: Ecological association between asbestos-related diseases and historical asbestos consumption: An international analysis. Lancet 369: 844-849, 2007.

4. Takahashi $\mathrm{K}$ and Landrigan PJ; Collequim Ramazzini: The global health dimensions of asbestos and asbestos-related diseases. Ann Glob Health 82: 209-213, 2016.

5. Tomasson K, Gudmundsson G, Briem H and Rafnsson V: Malignant mesothelioma incidence by nation-wide cancer registry: A population-based study. J Occup Med Toxicol 11: 37, 2016.

6. Scherpereel A, Astoul P, Baas P, Berghmans T, Clayson H, de Vuyst P, Dienemann H, Galateau-Salle F, Hennequin C, Hillerdal G, et al: Guidelines of the European Respiratory Society and the European Society of Thoracic Surgeons for the management of malignant pleural mesothelioma. Eur Respir J 35: 479-495, 2010.

7. Stahel RA, Weder W, Lievens Y and Felip E; ESMO Guidelines Working Group: Malignant pleural mesothelioma: ESMO clinical practice guidelines for diagnosis, treatment and follow-up. Ann Oncol 21 (Suppl 5): v126-v128, 2010.

8. van der Bij S, Schaake E, Koffijberg H, Burgers JA, de Mol BA and Moons KG: Markers for the non-invasive diagnosis of mesothelioma: A systematic review. Br J Cancer 104: 1325-1333, 2011.

9. Hollevoet K, Reitsma JB, Creaney J, Grigoriu BD, Robinson BW, Scherpereel A, Cristaudo A, Pass HI, Nackaerts K, Rodríguez Portal JA, et al: Serum mesothelin for diagnosing malignant pleural mesothelioma: An individual patient data meta-analysis. J Clin Oncol 30: 1541-1549, 2012.
10. Tanaka F, Yoneda $\mathrm{K}$ and Hasegawa S: Circulating tumor cell (CTC) in lung cancer: Current status and future perspectives. Lung Cancer (Auckl) 1: 77-84, 2010.

11. Tanaka $\mathrm{F}$ and Yoneda $\mathrm{K}$ : Adjuvant therapy following surgery in non-small cell lung cancer (NSCLC). Surg Today 46: 25-37, 2016.

12. Allard WJ, Matera J, Miller MC, Repollet M, Connely MC, Rao C, Tibbe AGJ, Uhr JW and Terstappen LW: Tumor cells circulate in the peripheral blood of all major carcinomas but not in healthy subjects or patients with nonmalignant diseases. Clin Cancer Res 10: 6897-6904, 2004.

13. Yoneda K, Tanaka F, Kondo N, Hashimoto M, Takuwa T, Matsumoto S, Okumura Y, Tsubota N, Sato A, Tsujimura T, et al: Circulating tumor cells (CTCs) in malignant pleural mesothelioma (MPM). Ann Surg Oncol 21 (Suppl 4): S472-S480, 2014.

14. Ohnaga T, Shimada Y, Moriyama M, Kishi H, Obata T, Takata K, Okumura T, Nagata T, Muraguchi A and Tsukada K: Polymeric microfluidic devices exhibiting sufficient capture of cancer cell line for isolation of circulating tumor cells. Biomed Microdevices 15: 611-616, 2013.

15. Chikaishi Y, Yoneda K, Ohnaga $T$ and Tanaka F: EpCAM-independent capture of circulating tumor cells with a 'universal CTC-chip'. Oncol Rep 37: 77-82, 2017.

16. Usami N, Fukui T, Kondo M, Taniguchi T, Yokoyama T, Mori S, Yokoi K, Horio Y, Shimokata K, Sekido Y and Hida T: Establishment and characterization of four malignant pleural mesothelioma cell lines from Japanese patients. Cancer Sci 97: 387-394, 2006

17. Kimura N and Kimura I: Podoplanin as a marker for mesothelioma. Pathol Int 55: 83-86, 2005.

18. Abe S, Morita Y, Kaneko MK, Hanibuchi M, Tsujimoto Y, Goto H, Kakiuchi S, Aono Y, Huang J, Sato S, et al: A novel targeting therapy of malignant mesothelioma using anti-podoplanin antibody. J Immunol 190: 6239-6249, 2013.

19. Ordóñez NG: D2-40 and podoplanin are highly specific and sensitive immunohistochemical markers of epithelioid malignant mesothelioma. Hum Pathol 36: 372-380, 2005.

20. Toss A, Mu Z, Fernandez S and Cristofanilli M: CTC enumeration and characterization: Moving toward personalized medicine. Ann Transl Med 2: 108, 2014.

21. Nagrath S, Sequist LV, Maheswaran S, Bell DW, Irimia D, Ulkus L, Smith MR, Kwak EL, Digumarthy S, Muzikansky A, et al: Isolation of rare circulating tumour cells in cancer patients by microchip technology. Nature 450: 1235-1239, 2007.

22. Husain AN, Colby TV, Ordóñez NG, Krausz T, Borczuk A, Cagle PT, Chirieac LR, Churg A, Galateau-Salle F, Gibbs AR, et al: Guidelines for pathologic diagnosis of malignant mesothelioma: A consensus statement from the international mesothelioma interest group. Arch Pathol Lab Med 133: 1317-1331, 2009.

23. Scales SJ, Gupta N, Pacheco G, Firestein R, French DM, Koeppen H, Rangell L, Barry-Hamilton V, Luis E, Chuh J, et al: An antimesothelin-monomethyl auristatin e conjugate with potent antitumor activity in ovarian, pancreatic, and mesothelioma models. Mol Cancer Ther 13: 2630-2640, 2014.

24. Sato A, Torii I, Okamura Y, Yamamoto T, Nishigami T, Kataoka TR, Song M, Hasegawa S, Nakano T, Kamei T and Tsujimura T: Immunocytochemistry of CD146 is useful to discriminate between malignant pleural mesothelioma and reactive mesothelium. Mod Pathol 23: 1458-1466, 2010. 